\title{
Angiogenic effects of cryosurgery with liquid nitrogen on the normal skin of rats, through morphometric study*
}

\author{
Camila Bianco Pimentel ${ }^{1}$ \\ Maria Letícia Cintra ${ }^{2}$ \\ DOI: http://dx.doi.org/10.1590/abd1806-4841.20142249
}

\begin{abstract}
BACKGROUND: Cryosurgery is an efficient therapeutic technique used to treat benign and malignant cutaneous diseases. The primary active mechanism of cryosurgery is related to vascular effects on treated tissue. After a cryosurgical procedure, exuberant granulation tissue is formed at the injection site, probably as a result of angiogenic stimulation of the cryogen and inflammatory response, particularly in endothelial cells.

OвJECTIVE: To evaluate the angiogenic effects of freezing, as part of the phenomenon of healing rat skin subjected to previous injury. MetHODS: Two incisions were made in each of the twenty rats, which were divided randomly into two groups of ten. After 3 days, cryosurgery with liquid nitrogen was performed in one of incisions. The rats' samples were then collected, cut and stained to conduct histopathological examination, to assess the local angiogenesis in differing moments and situations.

RESULTS: It was possible to demonstrate that cryosurgery, in spite of promoting cell death and accentuated local inflammation soon after its application, induces quicker cell proliferation in the affected tissue and maintenance of this rate in a second phase, than in tissue healing without this procedure.

CONCLUSIONS: These findings, together with the knowledge that there is a direct relationship between mononuclear cells and neovascularization (the development of a rich system of new vessels in injury caused by cold), suggest that cryosurgery possesses angiogenic stimulus, even though complete healing takes longer to occur. The significance level for statistical tests was $5 \%(p<0,05)$. Keywords: Cryosurgery; Neovascularization, physiologic; Skin ulcer; Wound healing
\end{abstract}

\section{INTRODUCTION}

Cryosurgery is a surgical technique that employs 20 freezing to destroy undesirable tissue. It is an efficient therapeutic technique to treat benign and malignant cutaneous diseases. ${ }^{1,2}$ The aim of cryosurgery is to cause necrosis of tissue. The length of application (i.e. the amount of freezing required) depends on the type of lesion. Biological changes occur as a result of rapid heat loss when cryogen touches the skin, in effect causing tissue destruction by freezing. ${ }^{3}$
Cryosurgery, sometimes referred to as cryotherapy or cryoablation, is an old and new technique, which has undergone a long-term process of development. There is evidence that cold was used in medicine as early as $3500 \mathrm{BC}$ to treat infected wounds. ${ }^{4}$

The ancient Egyptians, and later Hippocrates, were aware of the analgesic and anti-inflammatory properties of cold, which were used to treat infected lesions of the chest, fractures of the skull, and various 
battle injuries. Over the past 200 years, cold treatment has evolved from generalized application, such as hydrotherapy, to specific, focal destruction of tissue contemporary cryosurgery. ${ }^{5}$

Following a cryosurgical procedure, the formation of exuberant granulation tissue with marked vascular proliferation and wound-repair occurring with positive tissue recovery has been clinically observed. Despite the researchers' interest in the relationships between mechanisms that lead to tissue necrosis (cell death), particularly those related to vascular changes triggered by tissue freezing, few studies have evaluated the relationship between cryosurgery and local neovascularization. The loss of circulation and cellular anoxia are commonly considered the major mechanisms of injury in cryosurgery. The initial response to tissue freezing is vasoconstriction, which reduces blood flow, preventing circulation. When the tissue thaws and the temperature reaches $0^{\circ} \mathrm{C}$, circulation returns, but with vasodilation. Endothelial damage results in increased permeability of the walls of microcirculatory vessels, edema, platelet aggregation, and formation of microthrombi, leading to circulation stagnation in approximately 45 minutes. The loss of blood supply deprives all cells of any chance of survival and results in uniform tissue necrosis, with a border of preserved tissue. ${ }^{6}$

The release of blood constituents from damaged vessels begins the process of tissue healing. The tissue components that are involved in wound healing are: blood vessels, the epidermis, dermis, attachments (including hair and glands), nerves and subcutaneum. Consequently, the cell types involved mainly include endothelial cells, macrophages, neutrophils, platelets, epidermal keratinocytes, fibroblasts, outer root sheath cells, neurons and adipocytes. ${ }^{7}$

The destructive effects of freezing tissue through cryosurgery are due to a number of factors which can be grouped into two major mechanisms, one immediate, the other delayed. The immediate cause of injury is the deleterious effect of the cooling and warming cycles on the cells. After the tissue thaws, the delayed cause of injury begins, with the progressive failure of microcirculation and ultimate vascular stasis. The relative importance of these two mechanisms has long been debated with respect to frostbite. This debate continues in relation to cryosurgical injury, when it acts as a mechanism for injury. The complexity of the mechanism of injury in cryosurgery causes considerable difficulty in assessing the cause of injury in cryosurgical research in vivo.

Research in vitro does not provide information about the effect of changes in blood flow but is important in understanding cryogenic injury in relation to the direct deleterious effects of freezing upon cells. ${ }^{8}$
In 1956, Bellman and Adams-Ray demonstrated the vascular effects and resulting tissue damage after cold injury using microangiographic images. It was shown that cold trauma involving rapid freezing maintained 66 at a constant low temperature for ten minutes, followed by rapid thawing, injured the tissue less than similar cold trauma involving slow thawing. In addition, rapid formation of new blood vessels in injured tissue was confirmed. ${ }^{9}$

Situations in which post-embryonic angiogenesis occurs are frequent. Classic examples in physiological situations include the ovulation cycle (formation and regression of the corpus luteum) and the process of wound healing. Furthermore, several pathological conditions are associated with increased angiogenesis, such as inflammation, adaptation to ischemia, proliferative growth and the spread of tumors. ${ }^{8}$

Following a cold injury, the formation of new vessels is observed 24-48 hours after the trauma, with an ample amount of very fine blood vessels in the periphery of the lesion. After three to four days, larger blood vessels with a diameter of approximately $0.3 \mathrm{~mm}$ appear in the center of the lesion. New vessels have a serpiginous appearance, with most radiating from the periphery of the lesion in the surrounding tissue. Although it has been verified that small vessels can be damaged and permanently excluded from circulation, even when tissue subjected to cold has not died, the previously mentioned formation of new vessels proves that tissue may have sufficient nutrition through mere dissemination of vessels from the surrounding area to produce the rapid development of new blood vessels. ${ }^{7}$

Based on the results of various experiments, it was concluded that immediately following a cold injury, a permanent occlusion of small vessels will occur in the tissue, followed by the development of a rich system of new vessels between the involved tissue and outside the lesion, which will result in revascularization 89 of the affected area. ${ }^{7}$

The proliferation phase of the healing process is characterized by the formation of granulation tissue, which consists of new vessels that migrate into the lesion (angiogenesis/neovascularization), the accumulation of fibroblasts and dermal matrix, and endothelial cells that play the most important role in angiogenesis. ${ }^{6,8}$

Research on wound healing in rats using histopathological methods was done to evaluate, in a qualitative, quantitative or semiquantitative manner, the presence of blood vessels, fibroblasts, leukocytes (mononuclear and polymorphonuclear), reepithelization and collagen production. ${ }^{10}$ Published papers about immunohistochemistry, using specific markers to evaluate the expression of angiogenic factors, the 
number of pre-existing or newly formed vessels, as well as lymphatic vessels, also helped in determining local angiogenesis. ${ }^{11}$

This study sought to define the relationship between tissue damage by freezing with liquid nitrogen and the development of angiogenesis. Cryosurgery was performed on an experimental wound on the third 104 day of evolution, demonstrating its effect on the maintenance of cellularity, which has been proven to have a direct correlation to angiogenesis.

\section{MATERIAL AND METHODS}

Twenty young adult Lewis strain male rats were 107 used, weighing between 180 and 220 grams. These rats were divided randomly into two groups of 10 animals, group I (GI) and group II (GII), based on the time of the collection of samples for histopathological analysis. The rats were kept in individual cages with adequate food and water. Each rat received a cranial wound, which was established as the Control Area (CA) and a tail wound called the Treated Area (TA). The wounds were administered $2 \mathrm{~cm}$ apart using a $5 \mathrm{~mm}$ punch. After 3 days, the TA was subjected to cryosurgery with liquid nitrogen for 15 seconds, using the open spray technique. The CA was maintained without intervention.

After seven days (GI) and fourteen days (GII), samples from the two areas (CA and TA) were collected using an $8 \mathrm{~mm}$ punch (encompassing the entire lesion and $3 \mathrm{~mm}$ of surrounding tissue), immediately after the animals were euthanized with intravenous thiopental in the dorsal tail vein. The samples were fixed in $10 \%$ formalin for twelve hours and $70 \%$ alcohol for twelve additional hours, after which they were processed and embedded in paraffin. The paraffin blocks were cut $4 \mu$ thick, mounted on slides and stained with hematoxylin and eosin for use in histopathological analysis, using histometric counting of cell nuclei and morphological study.

A cycloid graticule attached to the eyepiece of an Olympus CBA microscope with 400x magnification (10x ocular, coupled with the objective of $40 \mathrm{x}$ immersion), was used for histometric counting. The definition of histometric fields was determined as 10 fields of systematic sampling in the upper dermis of the cell region, avoiding adjoining and necrotic structures, where all nuclei focused in the cycloids were counted.

A morphological study was conducted using 129 qualitative analysis of the extent of reepithelialization, the degree of residual depression and epidermal thickness. A clinical evaluation of the dimensions of the wounds, scabbing and degree of healing was also performed on day seven (GI) and day fourteen (GII).

\section{RESULTS}

These clinical observations were also demonstrated histologically. Cryosurgery promotes a very intense inflammatory process, greater than conventional inflammatory processes, and consequently generates greater vascular lesions and the formation of exuberant granulation tissue. The TA presented areas with larger lesions, persistent scabbing and increased healing time.

Due to the direct relationship between the quantity of mononuclear cells and newly formed vessels in tissue, mentioned by M. Calvin in 1998, and because of histological observations of a rapid increase in cellularity and its maintenance in the treated area of the present study, which was not observed in the control area, we are able to confirm that cryosurgery induces angiogenesis (Figure 1 and Graph 1).

\section{DISCUSSION}

Although studies showing the relationship between cryosurgery and angiogenic action are still rare, in 1956 Bellman and Adams-Ray showed the development of a rich system of new vessels in cold injuries.

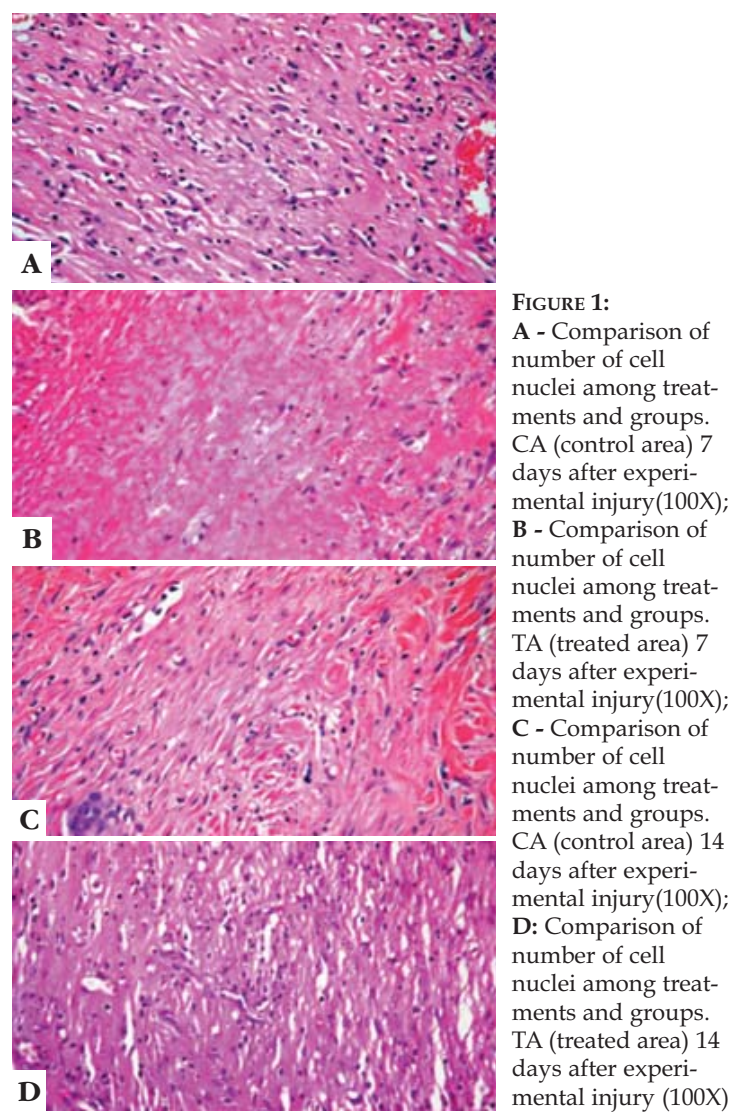




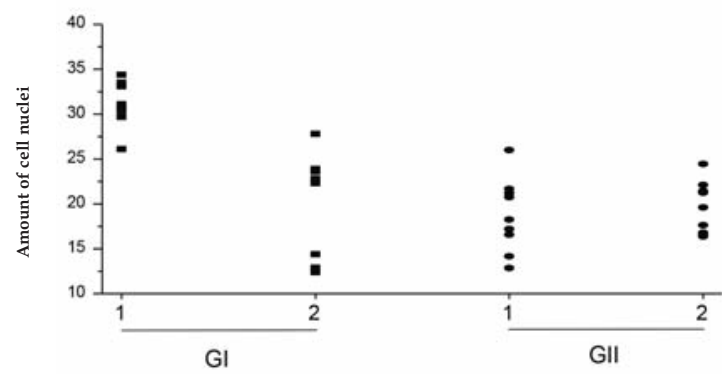

GRAPH 1: Graphic representation of the cellularity comparison in the two areas

An understanding of what occurs in pathological situations of post-fetal angiogenesis such as the healing of wounds, the inflammatory process, and ischemia adaptation (Carmeliet, P., 2003) further reinforces the local angiogenic effects of cryosurgery. The authors noted that, although the healing process takes longer, angiogenesis improves the overall quality when scarring is completed. Scarring resulting from an extensive period of freezing due to cryosurgery is hypochromic and slightly atrophic, while maintaining acceptable elasticity and recomposition of the extensive treated areas.

\section{CONCLUSION}

It is fitting to ask if exuberant granulation tissues can be absorbed after cryosurgery, as has been observed in other therapeutic procedures. Research of this type could also focus on the utilization of this method to promote angiogenesis in areas with insufficient microcirculation, such as in ischemic ulcers. Consequently, it is important that new studies using vascular markers following cryosurgery be carried out to ascertain the relationship between functional quantity and viability of newly formed vessels, with a view to the possible use of cryosurgery as an aid in treating illnesses with limited vascular support.]

\section{REFERENCES}

1. Lizhi N, Kecheng X, Feng M. Cryosurgery for lung câncer. J. Thorac. Dis. 2012; 4(4): 408-16.

2. Lucas R. Avaliação e comparação de diferentes técnicas de criocirurgia com nitrogênio líquido em cães da raça Beagle, pela tomografia computadorizada, histologia e imunohistoquímica [tese]. São Paulo (SP): Universidade de São Paulo; 2001.

3. Poziomczyk CS, Köche B, Dornelles Mde A, Dornelles SI. Pain evaluation in the cryosurgery of actinic keratoses. An Bras Dermatol. 2011;86:645-50.

4. Gage AA. History of cryosurgery. Semin Surg Oncol. 1998;14:99-109.

5. Gage AA, Baust J. Mechanisms of tissue injury in cryosurgery. Cryobiology. 1998;37:171-86.

6. Yamaguchi Y, Yoshikawa K. Cutaneous Wound healing: an update. J Dermatol. 2001;28:521-34.

7. Belman S, Adams-Ray J. Vascular reactions after experimental cold injury. Angiology. 1956; 7:339-67.

8. Carmeliet P. Angiogenesis in health and disease. Nat Med. 2003;9:653-60.

9. Calvin, M. Cutaneous wound repair. Wounds. 1998;10:12-32.

10. Fox SB, Harris AL. Histological quantitation of tumor angiogenesis. APMIS. 2004;112:413-30.

11. Fina L, Molgaard HV, Robertson D, Bradley NJ, Monaghan P, Delia D, et al. Expression of the CD34 gene in vascular endothelial cells. Blood. 1990;75:2417-26.

\author{
MAILING ADDRESS: \\ Camila Bianco Pimentel \\ Rua Coronel Quirino, 350 - Apt. 172 \\ Cambuí \\ 13025-000 Campinas, SP. \\ E-mail: cbpimentel.vet@gmail.com
}

How to cite this article: Pimentel CB, Moraes AM, Cintra ML. Angiogenic effects of cryosurgery with liquid nitrogen in normal skin rats, using morphometric study. An Bras Dermatol. 2014;89(3):410-3. 